\title{
More about the multiplicative model for the analysis of genotype-environment interaction
}

\author{
ROBERTO CRUZ M. \\ Instituto Tecnológico de Sonora, 5 de Febrero No. 818 Cd. Obregón, Son CP 8500, Mexico
}

In this paper various multiplicative models that are often used to analyse genotype-environment interaction are described and some of the statistical relationships between them are explored. It is shown that principal-component analysis can be seen as a natural generalization of the joint regression analysis. A set of maize data is used to exemplify these statistical models.

Keywords: joint regression, non-additivity general linear model, principal components.

\section{Introduction}

The most popular method to explain genotypeenvironment interaction seems to be the use of the multiplicative model. This model was first used by Mooers in 1921, as Westcott (1986) states, but Yates \& Cochran (1938) appear in most of the literature as the first users and Finlay \& Wilkinson (1963), Eberhart \& Russell (1966), Perkins \& Jinks (1968) as the authors who extended its use.

\section{The common multiplicative model}

Using the same model and notation as used by Perkins \& Jinks (1968) and Shukla (1972) in which $t$ genotypes are tested in a randomized complete block design with $r$ replications within each of $s$ environments we have the model:

$$
Y_{i j k}=\mu+d_{i}+\varepsilon_{j}+r_{j k}+g_{i j}+e_{i j k}
$$

where $\mu$ is the grand mean, $d_{i}(i=1,2, \ldots t)$ the additive genetic contribition of the $i$ th genotype, $\varepsilon_{j}(j=1,2, \ldots s)$ the additive environmental contribution of the $j$ th environment, $r_{j k}$ the additive contribution of the $k$ th block within the $j$ th environment $g_{i j}$ the genotype environmental interaction of the $i$ th genotype in the $j$ th environment and $e_{i j k}$ is the residual variation contributed by the $k$ th replicate of the $i$ th genotype in the $j$ th environment.

If we assume that blocks within environments, $\varepsilon_{j}$ and $d_{i}$ are fixed effects so that:

$\Sigma_{k} r_{i k}=0$

and as usual

$E\left(e_{i j k}\right)=0$ and $V\left(e_{i j k}\right)=\sigma^{2}$ then model (1) can be expressed in terms of the means $Y_{i j}$ (the mean of $r$ replicates of the $i$ th genotype at the $j$ th environment) as:

$Y_{i j}=\mu+d_{i}+\varepsilon_{j}+g_{i j}+e_{i j}$

Observe that restriction (2) is necessary for model (3).

Now if we assume the common multiplicative model:

$g_{i j}=b_{i} \varepsilon_{j}+\eta_{i j}$

and if we let

$\alpha_{i j}=\eta_{i j}+e_{i j}$ we have:

$Y_{i j}=\mu+d_{i}+\left(1+b_{i}\right) \varepsilon_{j}+\alpha_{i j}$

In this model the term $b_{i}$ can be interpreted as a sensitivity index that permits us to differentiate genotypes. If positive, $i$ th genotype has a better performance (with respect to the rest of the genotypes) in high yield environments than in low ones, if negative we have the contrary and if zero this genotype has an average sensitivity.

\section{The multiplicative model adjusted by regression}

The usual regression estimators of $b_{i}$ in model (5) are:

$\hat{b}_{i}=\Sigma_{j}\left(Y_{i j}-\bar{Y}_{. j}\right)\left(\bar{Y}_{. j}-\bar{Y}_{. .}\right) / \Sigma_{j}\left(\bar{Y}_{. j}-\bar{Y}_{. .}\right)^{2}$

or

$1+b_{i}=\Sigma_{j}\left(\bar{Y}_{. j}-\bar{Y}_{. .}\right) Y_{i j} / \Sigma_{j}\left(\bar{Y}_{. j}-\bar{Y}_{. .}\right)^{2}$.

The equality of all $b_{i}$ 's can be tested by calculating the sums of squares of non-additivity:

$\mathrm{SSNA}=r \Sigma_{i} b_{i}^{2} \Sigma_{j}\left(\bar{Y}_{. j}-\bar{Y}_{. .}\right)^{2}$

and the sum of squares of deviations of regression [or 
'balance' as named by Skukla (1972)].

SSDR $=$ SSGE - SSNA

where SSGE stands for the sum of squares of genotype-environment interaction:

Using the results of Mandel (1961), Shukla (1972), showed that the appropriate test for all $b_{i}=0$ is:

$F=\frac{\operatorname{SSNA} /(t-1)}{\operatorname{SSDR} /(t-1)(s-2)}$

As regression estimators have known conditional variances this allows the hypothesis $H_{0}: b_{i}=0$ to be tested for any genotype. This seems to be the main advantage of this method, but if regression estimators fail to explain genotype-environment interaction the fit can be improved by the bilinear approach.

\section{The multiplicative model adjusted by least squares}

Gabriel (1978) shows that a better fit of model (5) is obtained in two stages, in stage 1 a linear fit of $\mu$ and $d_{i}$ ignoring $\varepsilon_{j}$ is performed and in stage 2 a bilinear fit of $\left(1+b_{i}\right) \varepsilon_{j}$ to the residuals from the linear fit.

If $\varepsilon_{j}$ is considered as a fixed effect, the test proposed by Gabriel in which eigenvalues are used is not useful because we have in this case a non-central Wishart distribution, nevertheless $H_{0}: b_{i}=0$ can be proved with the likelihood ratio statistics (Appendix):

$-2 \ln \lambda=r s t \ln \left(1+\frac{r \lambda_{1}^{2}-\mathrm{SSE}}{\mathrm{SSe}}\right)$

where SSE is the sum of squares of environments and SSe is the sum of squares of error.

$H_{0}$ is rejected with an approximate $\alpha$-level if: $-2 \ln \lambda \geqslant \chi_{\alpha}^{2}$ with $t-1$ degrees of freedom where $\lambda_{1}$ is the largest eigenvalue in the singular value decomposition of the $t \times s$ order matrix $\mathbf{X}$ with elements:

$\mathbf{X}_{i j}=Y_{i j}-\bar{Y}_{i}$.

Let $q_{1}$ and $p_{1}$ be the eigenvectors of matrices $\mathbf{X X} \mathbf{X}^{\prime}$ and $\mathbf{X}^{\prime} \mathbf{X}$, respectively, corresponding with $\lambda_{1}$, where:

$q_{1}^{\prime}=\left(q_{11}, q_{12}, \ldots q_{1 t}\right)$

and

$p_{1}^{\prime}=\left(p_{11}, p_{12}, \ldots p_{1 s}\right)$.

The least squares estimators of $b_{i}$ are obtained in such a way that:

$\Sigma_{i}\left(1+b_{i}\right)=K \Sigma_{i} q_{1 i}=t$

and the estimators of $\varepsilon_{j}$ are:

$\hat{\varepsilon}_{j}=p_{1 j} / K$ or in equivalent form Shukla (1974)

$1+b_{i}=q_{1 i} / q_{m}$

where

$q_{m}=\Sigma_{i} q_{1 i} / t$.

This method gives a better fit of the multiplicative model than the one given by regression but the difference can be small. The sums of squares by this method are:

$\mathrm{SSNA}=r \lambda_{1}^{2}-\mathrm{SSE}$

and

SSR $=$ SSGE - SSNA.

Least squares estimators provide a better fit than regression estimators but exact inferential methods are not available to test $H_{0}: b_{i}=0$ for any genotype, then if the improvement in the fit is not great, regression estimates seem preferable.

\section{Generalization of the multiplicative model}

If we consider that:

$Y_{i j}=\mu+d_{i} f_{j}+\left(1+b_{i}\right) \varepsilon_{j}+\alpha_{i j}$

it is easily seen that model (5) is a particular case of model $(6)$ when $f_{j}=1$.

Model (6) can be fitted in the following way: in the first stage a linear fit of $\mu$ is made and then multiplicative terms are fitted by principal-component analysis. This is known not to be the least squares method (Gabriel, 1978) but this two-stage method can be useful to compare model (6) against model (5).

The sum of squares explained by two principal components is:

$\mathrm{SSTC}=r\left(\lambda_{1}^{2}+\lambda_{2}^{2}\right)$

and the sum of squares of non-additivity in this method is:

SSNA $=$ SSTC - SSE - SSG

$\lambda_{1}$ and $\lambda_{2}$ are the two largest eigenvalues in the singular value decomposition of the $t \times s$ order matrix $\mathbf{W}$ with elements

$W_{i j}=Y_{i j}-\bar{Y}_{\text {... }}$

The main advantage of principal components in this case, as mentioned before, is its graphical representation, then if $p_{1}$ is defined as above and $p_{2}$ in a similar way by now referring to matrix $\mathbf{W}$, the coordinates of the $i$ th genotype in the plane $Z_{1}-Z_{2}$ are:

$Z_{1 i}=\Sigma_{j} p_{1 j} W_{i j}$

$Z_{2 i}=\Sigma_{j} p_{2 j} W_{i j}$ 
This graphical representation of genotypes has an advantage with that given by Westcott (1987), it can be compared by its sum of squares with the common multiplicative model, the disadvantages of this approach is the inclusion of new parameters $\left(f_{i}: j=1,2\right.$, $\ldots s)$ and that inferential methods are not available.

Another generalization of model ( 5 ) is given by

$$
Y_{i j}=\mu+d_{i}+\varepsilon_{j}+b_{i} f_{j}+e_{i j}
$$

In this model, if $f_{j}=\varepsilon_{j}$ we have model (5). This model can be fitted by least squares (Gabriel, 1978) in two stages. In stage 1 a linear fit of $\mu, d_{i}$ and $e_{j}$ is required and in stage 2, a bilinear fit of $b_{i} f_{j}$ is made to the residuals from the linear fit.

An exact test of the bilinear term $b_{i} f_{j}$ is possible with percentage tables of eigenvalues of the Wishart matrix, for example the ones given by Johnson \& Graybill (1972) or Krzanowsky (1979). Unfortunately factor $f_{j}$ is usually difficult to interpret.

\section{Example}

In this example we analyse the data given by Acosta (1987) of 15 genotypes of maize in 13 environments in the north-west of México. In each environment a randomized complete-block design with four replicates was used.

The mean yield (ton/ha) of genotypes in each environment is given in Table 1 and Table 2 shows the analysis of variance in which genotype-environment interaction is found to be significant at the $\alpha=0.05$ level.

Table 1 Mean yield of genotypes by environments (ton/ha)

\begin{tabular}{|c|c|c|c|c|c|c|c|c|c|c|c|c|c|}
\hline \multirow[b]{2}{*}{ Genotypes } & \multicolumn{13}{|c|}{ Environments } \\
\hline & 1 & 2 & 3 & 4 & 5 & 6 & 7 & 8 & 9 & 10 & 11 & 12 & 13 \\
\hline 1 & 5.495 & 5.397 & 3.138 & 3.219 & 2.195 & 8.027 & 3.792 & 3.822 & 4.788 & 3.863 & 6.768 & 3.515 & 3.925 \\
\hline 2 & 7.775 & 4.619 & 4.902 & 2.469 & 3.941 & 10.669 & 3.798 & 3.132 & 6.409 & 3.363 & 8.704 & 3.426 & 4.825 \\
\hline 3 & 8.050 & 5.723 & 4.578 & 2.440 & 4.183 & 10.529 & 4.043 & 4.055 & 6.237 & 4.777 & 9.074 & 3.832 & 4.575 \\
\hline 4 & 7.000 & 3.928 & 5.232 & 2.337 & 3.594 & 9.356 & 3.347 & 3.598 & 5.879 & 4.328 & 8.209 & 3.561 & 2.350 \\
\hline 5 & 7.325 & 4.337 & 5.016 & 1.982 & 3.659 & 8.070 & 2.945 & 3.587 & 6.172 & 5.515 & 8.357 & 3.866 & 4.525 \\
\hline 6 & 6.925 & 4.637 & 5.030 & 2.711 & 2.306 & 8.232 & 3.571 & 3.704 & 5.878 & 3.714 & 5.572 & 3.060 & 4.025 \\
\hline 7 & 7.650 & 5.918 & 4.441 & 3.496 & 2.899 & 9.017 & 3.986 & 4.901 & 5.525 & 3.002 & 5.987 & 3.717 & 4.125 \\
\hline 8 & 7.800 & 4.281 & 5.304 & 2.972 & 1.301 & 8.730 & 4.349 & 4.523 & 5.342 & 4.206 & 6.057 & 4.351 & 4.250 \\
\hline 9 & 8.125 & 4.906 & 5.811 & 1.535 & 3.908 & 9.154 & 3.091 & 2.785 & 6.488 & 4.219 & 7.414 & 4.042 & 5.050 \\
\hline 10 & 8.225 & 4.402 & 5.140 & 1.533 & 3.891 & 8.882 & 4.004 & 2.816 & 5.204 & 5.027 & 8.556 & 3.683 & 5.750 \\
\hline 11 & 7.425 & 4.815 & 6.903 & 2.223 & 1.549 & 11.388 & 4.037 & 5.409 & 7.271 & 2.160 & 8.695 & 3.583 & 4.975 \\
\hline 12 & 6.025 & 4.185 & 4.569 & 2.704 & 2.959 & 6.200 & 3.112 & 3.532 & 6.128 & 2.369 & 4.303 & 3.275 & 3.775 \\
\hline 13 & 4.800 & 4.192 & 5.666 & 0.695 & 1.034 & 4.109 & 2.560 & 1.303 & 4.530 & 5.679 & 3.948 & 1.964 & 3.735 \\
\hline 14 & 6.050 & 3.256 & 4.665 & 0.814 & 2.204 & 7.434 & 2.592 & 1.274 & 4.505 & 2.435 & 7.814 & 1.572 & 3.450 \\
\hline 15 & 8.775 & 5.685 & 4.472 & 2.732 & 1.112 & 9.978 & 3.573 & 2.811 & 4.961 & 5.044 & 7.559 & 4.250 & 4.975 \\
\hline $\bar{Y}_{. j}$ & 7.193 & 4.685 & 4.925 & 2.257 & 2.716 & 8.653 & 3.520 & 3.417 & 5.668 & 3.980 & 7.133 & 3.446 & 4.287 \\
\hline
\end{tabular}

Table 2 Analysis of variance

\begin{tabular}{lrrrrl}
\hline Source & \multicolumn{1}{l}{ d.f. } & \multicolumn{1}{l}{ SS } & \multicolumn{1}{l}{ MS } & \multicolumn{1}{l}{$F_{\mathrm{c}}$} & $F_{a=0.05}$ \\
\hline Environments & 12 & 2636.910 & 219.742 & $46.21^{*}$ & 1.75 \\
Replications & 39 & 56.953 & 1.460 & & \\
Genotypes & 14 & 288.570 & 20.612 & $26.53^{*}$ & 1.70 \\
G-E & 168 & 555.957 & 3.309 & $4.26^{*}$ & 1.22 \\
NA & 14 & 141.333 & 10.095 & $3.75^{*}$ & 1.65 \\
DR & 154 & 414.624 & 2.692 & $3.46^{*}$ & 1.22 \\
Error & 546 & 424.175 & 0.777 & & \\
Total & 779 & 3962.565 & & & \\
\hline
\end{tabular}


Table 3 Coefficients and coordinates of genotypes

\begin{tabular}{|c|c|c|c|c|c|}
\hline \multirow[b]{2}{*}{ Genotype } & \multirow{2}{*}{$\begin{array}{l}\text { Mean yield } \\
\text { (ton/ha) }\end{array}$} & \multicolumn{2}{|l|}{ Coefficients } & \multicolumn{2}{|c|}{ Coordinates } \\
\hline & & Regression & Least Squares & $Z_{1}$ & $Z_{2}$ \\
\hline 3 & 5.545 & 0.1852 & 0.1893 & 8.034 & 2.425 \\
\hline 11 & 5.418 & 0.3814 & 0.3871 & 9.316 & 2.272 \\
\hline 2 & 6.233 & 0.2483 & 0.2554 & 8.406 & 1.537 \\
\hline 10 & 5.162 & 0.0950 & 0.0978 & 7.344 & 0.522 \\
\hline 9 & 5.117 & 0.1177 & 0.1165 & 7.459 & 0.362 \\
\hline 15 & 5.071 & 0.2233 & 0.2188 & 8.127 & 0.285 \\
\hline 5 & 5.027 & -0.0267 & -0.0267 & 6.493 & 0.096 \\
\hline 7 & 4.974 & -0.1384 & -0.1385 & 5.745 & 0.990 \\
\hline 8 & 4.882 & -0.0858 & -0.0888 & 6.052 & 0.112 \\
\hline 4 & 4.824 & 0.0716 & 0.0863 & 7.201 & 0.095 \\
\hline 6 & 4.566 & -0.1298 & -0.1338 & 5.690 & -1.052 \\
\hline 1 & 4.492 & -0.2184 & -0.2161 & 5.131 & -0.769 \\
\hline 12 & 4.089 & -0.4135 & -0.4171 & 3.715 & -2.377 \\
\hline 14 & 3.697 & 0.1326 & 0.1376 & 7.301 & -4.078 \\
\hline 13 & 3.323 & -0.4520 & -0.4676 & 3.211 & -6.714 \\
\hline
\end{tabular}

\section{The common multiplicative model adjusted by regression}

The mean yield of genotypes and their regression coefficients are given in Table 3. In this table it can be seen that:

$$
\begin{aligned}
& \mathrm{SSNA}=4(0.80393)(43.9507)=141.3331 \\
& \mathrm{SSDR}=555.9570-141.3331=414.6239 .
\end{aligned}
$$

The exact test of the multiplicative model is given below the G-E interaction line in Table 2, there it can be seen that both the multiplicative model and the deviations of regression are found to be significant at $\alpha=0.05$. In order to improve the adjustment, the bilinear fit was tried.

\section{Multiplicative model adjusted by least squares}

After calculating matrix $\mathbf{X}$ with elements $X_{i j}=Y_{i j}-\bar{Y}_{i}$, we obtain $\lambda_{1}=26.3670, \Sigma \lambda_{i}^{2}=798.1848$.

$$
\begin{aligned}
p_{1}^{\prime}= & (0.3577,-0.0220,0.0220,-0.3736,-0.2977, \\
& 0.5941,-0.1856,-0.1993,0.1295,-0.1312, \\
& 0.3724,-0.1948,-0.714), \\
q_{1}^{\prime}= & (0.1970,0.3155,0.2989,0.2730,0.2446, \\
& 0.2177,0.2165,0.2290,0.2806,0.2759,0.3486, \\
& 0.1465,0.1338,0.2859,0.3063) .
\end{aligned}
$$

From this

$$
q_{m}=\Sigma_{i} q_{1 i} / t=0.25132
$$

and

$1+b_{1}=q_{11} / q_{m}=0.1970 / 0.25132=0.7838$

or

$b_{1}=-0.2161$.

In a similar way we obtain the rest of $b_{i}$, which are listed in Table 3 . It can be seen from Table 3 that regression and least squares coefficients are very similar and the sum of squares of non-additivity is:

$$
\begin{aligned}
\text { SSNA } & =r \lambda_{1}^{2}-\mathrm{SSE}=4 \times 695.2188-2636.9100 \\
& =143.9653 .
\end{aligned}
$$

It can be seen that:

$$
\begin{aligned}
-2 \operatorname{In} \lambda & =4 \times 15 \times 13 \ln (1+143.9652 / 424.175) \\
& =227.93
\end{aligned}
$$

and

$-2 \ln \lambda>\chi_{0.05}^{2}=21$

with 12 d.f.

The hypothesis of equality of least squares coefficients is rejected but, as coefficients are very similar and SSNA is only $2 \%$ larger than that given by the regression approach, the gain is poor and we will try the generalization of a multiplicative model to improve the fit.

\section{Generalization of the multiplicative model}

After calculating matrix $\mathbf{W}$ with elements $W_{i j}=Y_{i j}-\bar{Y}_{.}$, we obtain vectors $\mathbf{p}_{1}$ and $\mathbf{p}_{2}$, which are shown in Table 
Table 4 Principal components

\begin{tabular}{lrrl}
\hline Environments & $\bar{Y}_{. j}-\bar{Y}_{. .}$ & \multicolumn{1}{l}{$p_{1}$} & \multicolumn{1}{l}{$p_{2}$} \\
\hline 1 & 2.431 & 0.3729 & 0.1761 \\
2 & -0.077 & -0.0064 & 0.1705 \\
3 & 0.163 & 0.0371 & 0.0882 \\
4 & -2.504 & -0.3564 & 0.3732 \\
5 & -2.046 & -0.2805 & 0.3583 \\
6 & 3.892 & 0.6102 & 0.3885 \\
7 & -1.242 & -0.1693 & 0.2646 \\
8 & -1.345 & -0.1819 & 0.4414 \\
9 & 0.927 & 0.1447 & 0.1423 \\
10 & -0.781 & -0.1160 & 0.0123 \\
11 & 2.372 & 0.3882 & 0.2731 \\
12 & -1.316 & -0.1777 & 0.3435 \\
13 & -0.475 & -0.0554 & 0.1890 \\
\hline
\end{tabular}

4. From this table it can be seen that the signs of elements of vector $\mathbf{p}_{1}$ are equal to those of the difference $\bar{Y}_{. j}-\bar{Y}_{\text {.. }}$ and so vector $\mathbf{p}_{1}$ can be interpreted as a comparison between environments of high yield with environments of low yield. Vector $\mathbf{p}_{2}$ can be interpreted as an average yield since its elements are all positive.

The two largest eigenvalues of matrix $\mathbf{W}$ are:

$\lambda_{1}=26.407, \lambda_{2}=9.162$ and $\Sigma_{i} \lambda_{i}^{2}=870.011$.

Observe that:

$r \Sigma_{i} \lambda_{i}^{2}=\mathrm{SSE}+\mathrm{SSG}+\mathrm{SSGE}=3481.437$

and

$\mathrm{SSNA}=r\left(\lambda_{1}^{2}+\lambda_{2}^{2}\right)-\mathrm{SSE}-\mathrm{SSG}=199.608$.

This sum of squares of non-additivity is $40 \%$ larger than that given by regression and least squares methods and it explains $36 \%$ of the sum of squares of the genotype-environment interaction.

We need the coordinates of genotypes for a graphical representation; for genotype 1 we have:

$$
\begin{aligned}
Z_{11}= & \Sigma_{j} p_{1 j} W_{1 j}=0.3729 \times 1.1884+\ldots \\
& +(-0.554) \times(-0.8366)=5.1310 \\
Z_{21}= & \Sigma_{j} p_{2 j} W_{i j}=0.1761 \times 1.1884+\ldots \\
& +0.1890 \times(-0.8366)=-0.7691 .
\end{aligned}
$$

Coordinates for the rest of the genotypes are calculated in a similar way (Table 3 ) observe the exceptional corresponding trends between means, coefficients and coordinates.

In Fig. 1 genotypes are represented by two principal components, the scale of component two is $\lambda_{2} / \lambda_{1}$ times the scale of component one in order to take into account the difference in the sum of squares explained by these components.

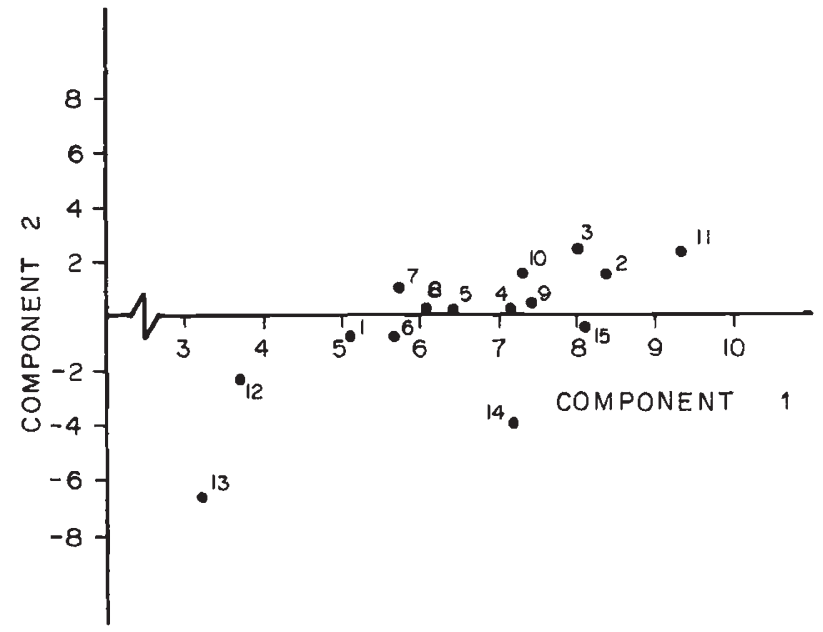

Fig. 1. Plot of the first two principal components of 15 maize genotypes.

In this figure the magnitude of yield corresponds to the coordinate of component one and the absolute value of the coordinate of component two corresponds to the difference in performance between high yield and low yield environments, if component two is positive then genotype performance is better in high yield than in low yield environments. Thus genotypes 2,3 and 11 are the genotypes of highest yield and largest difference in yield between high and low yield environments.

We can conclude that, in this example, the generalization of the multiplicative model is useful to explain genotype-environment interactions.

\section{References}

AcostA, B. J. A. 1987. Evaluacion y estabilidad de 15 variedades de maiz (Zea mays l.) en 13 localidades del noroeste de México. Tesis profesional, Universidad Autónoma Chapingo, México.

EBERHART, S. A. AND RUSSELL, W. A. 1966. Stability parameters for comparing varieties. Crop Sci., 6, 36-40.

FINLAY, K. W. AND WILKINSON, G. N. 1963. The analysis of adaptation in a plant breeding program. Aust. J. Agric. Res., 14, 742-754.

GABRIEL, K. R. 1978. Least squares approximation of matrices by additive and multiplicative models. J. R. Statis. Soc. B, 40, 186-196.

JOHNSON, D. E. AND GRAYBILL, F. A. 1972. Estimation of $\sigma^{2}$ in a two way classification model with interaction. J. Am. Stat. Assoc., 67, 862-868.

KRZANOWSKY, W. J. 1979. Some exact percentage points of a statistics useful in analysis of variance and principal component analysis. Technometrics, 21, 261-263.

MANDEL, J. 1961. Non-additivity in two way analysis of variance. J. Am. Stat. Assoc., 56, 878-888. 
PERKINS, J. M. AND JINKS, J. L. 1968. Environmental and genotype environmental components of variability. Heredity, 30, 111-126.

SHUKLA, G. K. 1972. Some statistical aspects of partitioning genotype environmental components of variability. Heredity, 29, 237-245.

SHUKLA, G. K. 1974. Variability in variety trials. PhD Thesis University of Edinburgh, Scotland, U.K.

WESTCOTT, B. 1986. Some methods of analysing genotypeenvironment interaction. Heredity, 56, 243-253.

WESTCOTT, B. 1987. A method of assessing the yield stability of crop genotypes. J. Agric. Sci., 180, 267-274.

YATES, F. AND COCHRAN, w. G. 1938. The analysis of group experiments. J. Agric. Sci., 28, 556-580.

\section{Appendix}

In this section model $(1)$ is tested with the likelihood ratio test against model (5). When this last model is referred to individual observations we have:

$Y_{i j k}=\mu+d_{i}+\left(1+b_{i}\right) \varepsilon_{j}+r_{i j}+e_{i j k}$.

In model (1) parameters $\mu_{i j k}=d_{i}+\varepsilon_{j}+r_{j k}+g_{i j}$ with the usual conditions:

$\Sigma d_{i}=0, \Sigma \varepsilon_{j}=0, \Sigma_{k} r_{j k}=0, \Sigma_{i} g_{i j}=0, \Sigma_{j} g_{i j}=0$

belong to parametrical space $\Omega$, it can be shown that the likelihood function $\ln L_{\Omega}$ is: $\ln L_{\Omega}=-(r s t / 2) \ln 2 \pi-(r s t / 2) \ln (\mathrm{SSe} / r s t)-r s t / 2$

because the least value of:

$\Sigma\left(Y_{i j k}-\mu_{i j k}\right)^{2}$

is given by the sum of squares of error SSe in the analysis of variance table.

In model (A1) parameters $\mu_{i j k}=d_{i}+\varepsilon_{j}+r_{j k}+b_{i} \varepsilon_{j}$ with the additional restriction $g_{i j}=b_{i} \varepsilon_{j}$ belonging to space $\omega$. In order to obtain the likelihood function $\ln L_{\omega}$ in this space, observe that the least value of $\Sigma\left(Y_{i j k}-g_{i j}\right)^{2}$ is given by $\Sigma\left(Y_{i j k}-Y_{i j}\right)^{2}$ because:

$\Sigma\left(Y_{i j k}-g_{i j}\right)^{2}=\Sigma\left(Y_{i j k}-\bar{Y}_{i j}\right)^{2}+r\left(\bar{Y}_{i j}-g_{i j}\right)^{2}$

so that the least squares estimators of $g_{i j}=\left(1+b_{i}\right) \varepsilon_{j}$ may be obtained just with means $\bar{Y}_{i j}$ and the results of Gabriel (1978) can be supplied. These estimators are given above and the likelihood function $\ln L_{\omega}$ is:

$\ln L_{\omega}=-(r s t / 2) \ln 2 \pi$

$$
\left.-(r s t / 2) \operatorname{In}\left[\left(r \lambda_{1}^{2}+\mathrm{SSe}-\mathrm{SSE}\right) / r-s t\right)\right]-r s t / 2
$$

and the likelihood ratio statistic for testing model (1) against model (A1) is:

$-2 \ln \lambda=r s t \ln \left[1+\left(r \lambda_{1}^{2}-\mathrm{SSE}\right) / \mathrm{SSe}\right]$

which has approximately a $\chi^{2}$ distribution with $t-1$ d.f. 